\title{
Effect of laser irradiance on the surface morphology and laser induced plasma parameters of zinc
}

\author{
MAHREEN AKRAM, SHAZIA BASHIR, ASMA HAYAT, KHALIQ MAHMOOD, RIAZ AHMAD, AND \\ M. KHALEEQ-U-RAHAMAN \\ Centre for Advanced Studies in Physics, Govt. College University, Lahore, Pakistan \\ (Received 5 September 2013; AcCEPTEd 22 November 2013)
}

\begin{abstract}
The effect of laser-irradiance on the surface morphology and laser induced breakdown spectroscopy of zinc has been investigated by employing Nd:YAG laser (wavelength $\lambda=1064 \mathrm{~nm}$, pulse duration $t \sim 10 \mathrm{ns,} \mathrm{and} \mathrm{repetition} \mathrm{rate}=$ $10 \mathrm{~Hz}$ ) under ambient environment of argon at a pressure of 20 Torr. For this purpose, zinc targets were exposed to various laser irradiances ranging from $13 \mathrm{GW} / \mathrm{cm}^{2}$ to $100 \mathrm{GW} / \mathrm{cm}^{2}$. Scanning electron microscope analysis has been performed to analyze the surface modification of irradiated zinc targets. Scanning electron microscope analysis revealed the formation of various kinds of structures such as ripples, cones, cavities, and wave like ridges at the center and peripheral regions of ablated zinc. In the central ablated region with increasing laser irradiance, the growth of distinct and well defined ripples is observed. Further increase in irradiance makes the appearance of these ripples diffusive and narrow. In order to correlate the plasma parameters with the surface modification, laser induced breakdown spectroscopy analysis has also been performed. The electron temperature and number density of zinc plasma have been evaluated at various laser irradiances. For both plasma parameters, an increasing trend up to a certain value of laser irradiance is observed which is due to enhanced energy deposition. Afterword a decreasing trend is achieved which is attributed to the shielding effect. With further increase in irradiance a saturation stage comes and almost no change in plasma parameters is observed. This saturation is explainable on the basis of the formation of a self-regulating regime near the target surface. A strong correlation between surface modification and plasma parameters is established.
\end{abstract}

Keywords: Electron density; Electron temperature; Laser-induced breakdown spectroscopy; Pulsed laser ablation; Surface structuring; Zinc

\section{INTRODUCTION}

Laser ablation is being widely used for surface structuring, chemical processing, and growth of thin films of a variety of materials. Textured surfaces with micro- and nano-scale morphological features show significantly altered physical, electrical, and mechanical properties (Latif et al., 2012; Jelani et al., 2013). A laser initiates a variety of processes upon interaction with the material through various energy transfer mechanisms depending on the irradiation conditions (Crouch et al., 2004). Observation of different mass removal regimes during the laser ablation of an aluminum target in air has been investigated by Cristoforetti et al. (2008). Comparing the trends of line intensity of the atomized plasma mass and the crater volumes, four laser fluence ranges were identified where

Address correspondence and reprint requests to: Mahreen Akram, Centre for Advanced Studies in Physics, Govt. College University, Lahore 54000, Pakistan. E-mail: shaziabashir@gcu.edu.pk different mass removal mechanisms seem to occur. Various potential material removal mechanisms have been discussed together with their applicability for short pulse laser ablation of metallic matrices by Bleiner et al. (2006). Lasers can induce topographical modifications through direct thermal ablations, optical interference patterning, splashing, spinodal breakdown, and hydrodynamic mechanisms (Crouch et al., 2004; Bleiner et al., 2006). A substantial work has been reported on the pulsed laser induced micro- and nano-structuring of various kinds of materials such as metals, semiconductors, dielectrics etc. (Sobhani et al., 2013; Bashir et al., 2012b). Laser induced periodic surface structures, usually referred as ripples, are the commonly observed structures. Their formation mechanism is still not fully understood although a number of theories have been presented by various research groups (Crouch et al., 2004).

A substantial amount of work is reported on laser irradiation effects on various metals (Butt et al., 2013; 
Trtica \& Stasic et al., 2013). But a very few work is reported on the laser induced nano-structuring for low melting point metals like zinc. Recently, the effect of laser focusing conditions on the surface modification and plasma parameters for zinc under ambient environment of vacuum has been studied. The formation of regular periodic surface structures by multi-pulse laser irradiation of zinc under vacuum condition has also been reported (Chen et al., 2012a; 2012b).

The nanosecond laser ablation of materials gives a dense and strongly ionized plasma plume of the ablated species due to laser interaction with the target and plume (Shaikh et al., 2006b). Laser generated plasmas are technically important and have wide range of applications in ablation, deposition, and laser based ion sources (Chamorro et al., 2012). The plasma parameters such as electron temperature and density have been investigated as a function of various experimental variables, e.g., laser irradiance, nature, and pressure of ambient gas etc. (Shaikh et al., 2006b; Hanif et al., 2013). The optimization of these parameters helps to understand the complex ablation mechanisms and control growth of structures (Sarkar et al., 2011). The presence of plasma strongly affects the ablation process and plays a key role for surface modification of the irradiated target. The ions/neutrals with known energy and flux can be applicable for laser assisted ion-sources and thin film growth (Ilyas et al., 2012).

The confinement and shielding effect (Farid et al., 2012; Cristoforetti et al., 2008; Cabalín et al., 1999; Bleiner et al., 2006), enhanced chemical reactivity along mechanical action (Hermann et al., 1993) and additional energies produced by laser supported heat, shock, and combustion waves (Bäuerle et al., 2011) of plasma effectively participate for the growth of various kinds of structures. The effect of nature and pressure of ambient gas on laser induced plasma temperature and number density, the surface morphology of the irradiated metallic targets and co-relation between them has been investigated (Khan et al., 2013; Bashir et al., 2012a). In the same context, generation and evolution of femtosecond laser induced silicon plasma by using time resolved spectroscopy along with the surface modification has been studied in various ambient gases at different laser fluences (Chen et al., 2012c).

Surface modification of zinc at micro- and nano-scale can enhance its optical absorption, thermionic and field emission properties of structured metal. The obtained results can also be applied for laser assisted deposition of metallic zinc films and implantation of zinc ions (Juan et al., 2013).

The aim of the present work is to investigate the effect of laser irradiance on the surface morphology of the irradiated zinc targets under ambient environment of argon. For this purpose, zinc targets have been exposed to various laser irradiances ranging from $13 \mathrm{GW} / \mathrm{cm}^{2}$ to $100 \mathrm{GW} / \mathrm{cm}^{2}$. The resulting surface modification has been analyzed using scanning electron microscope (SEM). In order to explore the effect of plasma on the ablation mechanisms, Laserinduced breakdown spectroscopy (LIBS) analysis has been also performed. The electron temperature and number density of the zinc plasma have been evaluated for various laser irradiances. SEM analysis has been correlated with the LIBS investigations.

\section{EXPERIMENTAL DETAILS}

A Nd:YAG laser (CRF200: Big Sky Laser Technologies, Quantel, France) with the central wavelength of $1064 \mathrm{~nm}$, pulse duration of $10 \mathrm{~ns}$, maximum pulse energy of $200 \mathrm{~mJ}$, at a repetition rate of $1-10 \mathrm{~Hz}$ has been employed to perform the ablation and emission spectroscopy of zinc. Rectangular sheets of zinc with dimensions of $3 \times 15 \times 10 \mathrm{~mm}^{3}$ were used as target. Prior to laser treatment samples were grinded, polished, and ultrasonically cleaned with acetone for $30 \mathrm{~min}$. Targets were placed in a stainless steel vacuum chamber, which was evacuated to a residual base pressure of about $10^{-3}$ mbar with the help of a rotary pump and then argon was filled at a pressure of 20 Torr. A lens of $50 \mathrm{~cm}$ focal length was used to focus the beam at right angle to the target surface with a spot size of $75 \mathrm{um}$. Two set of experiments were performed. In the first part, the targets were exposed to 200 laser pulses for SEM analysis, at four laser irradiances of $25 \mathrm{GW} / \mathrm{cm}^{2}, 50 \mathrm{GW} / \mathrm{cm}^{2}, 75 \mathrm{GW} / \mathrm{cm}^{2}$, and $100 \mathrm{GW} / \mathrm{cm}^{2}$ corresponding to laser pulsed energies of $50 \mathrm{~mJ}, 100 \mathrm{~mJ}, 150 \mathrm{~mJ}$, and $200 \mathrm{~mJ}$, respectively. The second set of experiment was performed for LIBS analysis. For this purpose, targets were exposed to single laser shot for laser irradiances ranging from $13 \mathrm{GW} / \mathrm{cm}^{2}$ to $100 \mathrm{GW} / \mathrm{cm}^{2}$.

The basic theme of the paper is to discuss the effects of plasma parameters on the laser-induced surface structuring of zinc. In this regards, the role of excitation temperature and electron density on the surface structuring of zinc has been addressed. The LIBS analysis is usually performed with single shot experiment. When SEM analysis of zinc with single pulse irradiation is performed no significant effects on the surface can be explored. Therefore samples were exposed with 200 numbers of overlapping pulses and then the corresponding surface modification is correlated with the accumulated effects of plasma parameters.

All targets (for both SEM and LIBS analysis) were exposed at a distance of $3 \mathrm{~mm}$ before the focus. This configuration was used to minimize the breakdown in the corresponding gas. The Surface morphology of the irradiated targets was investigated by using SEM (JEOL JSM-6480 LV). Emission spectra were collected by a LIBS2500 plus spectrometer system (Ocean Optics).

\section{RESULTS AND DISCUSSION}

\section{SEM Analysis}

SEM analysis reveals the surface modification of the zinc and illustrates the formation of craters for all fluences. However, an image of Figure 1a is an example which reveals the overall 
a)

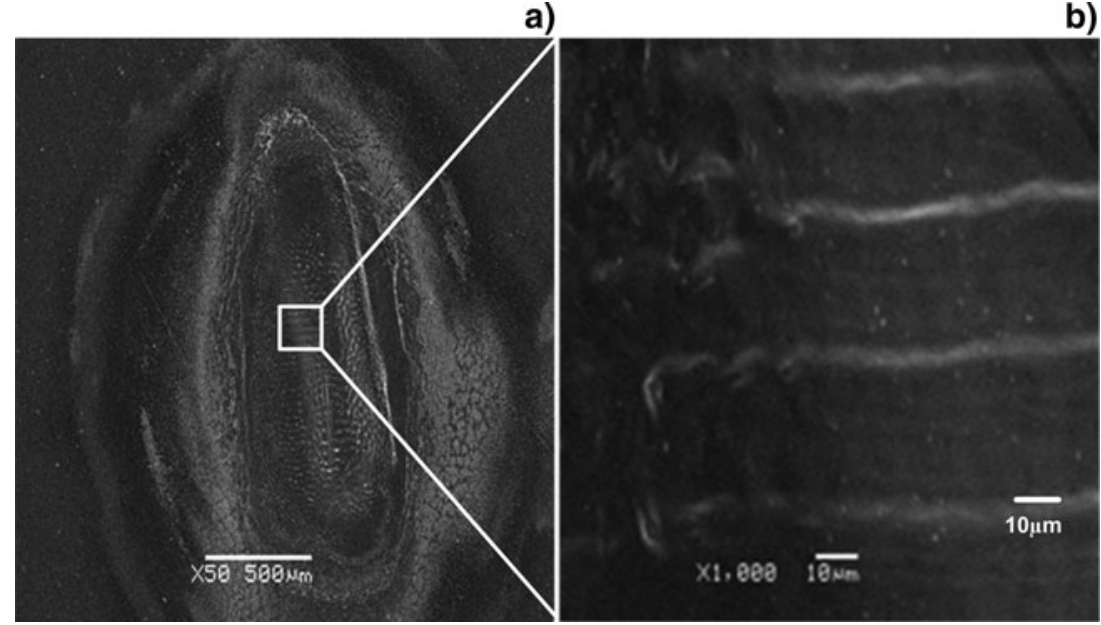

b)
Fig. 1. SEM images revealing the surface modification of zinc at laser irradiance of $100 \mathrm{GW} / \mathrm{cm}^{2}$. (a) The overall modified surface of zinc after laser ablation with crater and ripple- formation. (b) The magnified view of the central ablated area with only an appearance of ripples. modified surface of zinc (crater and ripple - formation) after ablation at a laser irradiance of $100 \mathrm{GW} / \mathrm{cm}^{2}$ corresponding to pulse energy of $200 \mathrm{~mJ}$. The whole crater is displayed (showing the crater + part of un-ablated surface) in Figure 1a. Whereas Figure $1 \mathrm{~b}$ exhibits the magnified view of the central ablated area of zinc (inset of Fig. 1a) in which only ripples are seen.
SEM images of Figure 2 illustrate the effect of laser irradiance on the surface morphology of the irradiated zinc targets. Figure $2 \mathrm{a}-2 \mathrm{~d}$ represents the central ablated region. It is observed that laser irradiated surfaces are corrugated and rippled at central ablated areas. Figure 2a shows the growth of indistinct ripples for the lowest irradiance of $25 \mathrm{GW} / \mathrm{cm}^{2}$. When

a)

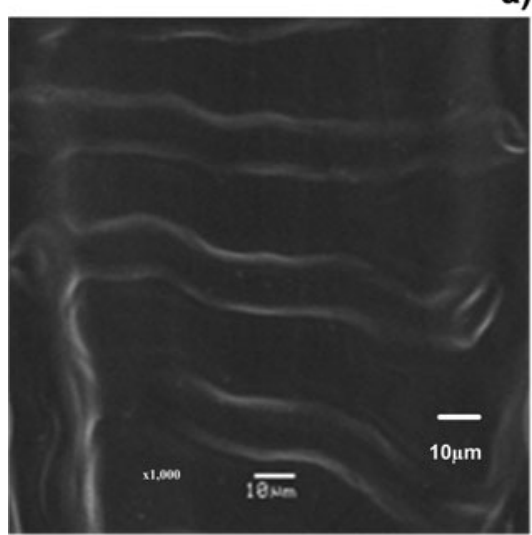

c)

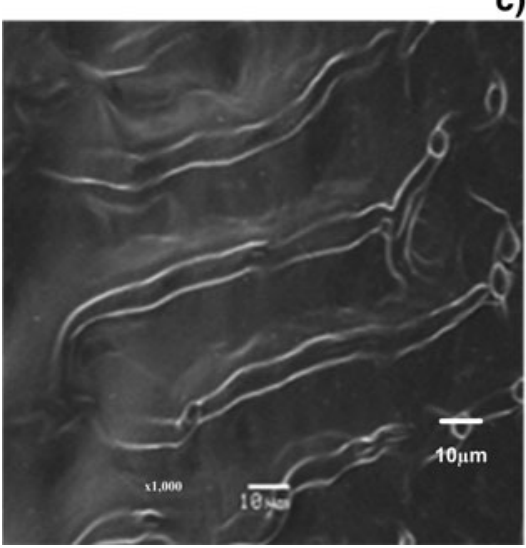

d)

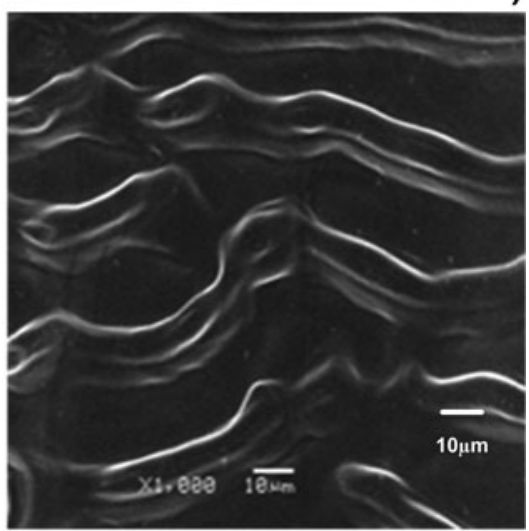

d)

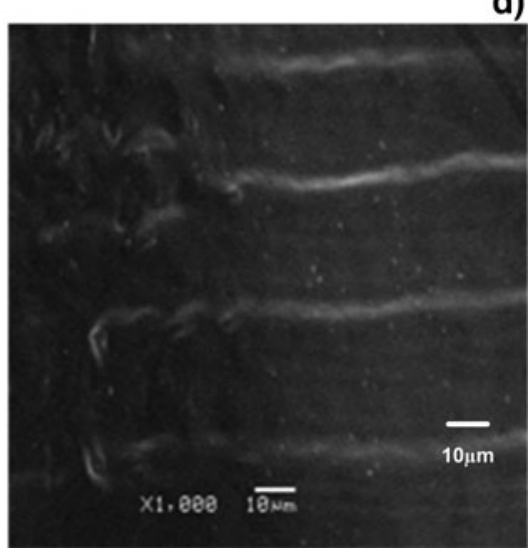

Fig. 2. SEM images of the central ablated region of zinc targets at various laser irradiances of (a) 25 , (b) 50 , (c) 75 , and (d) $100 \mathrm{GW} / \mathrm{cm}^{2}$. 
the irradiance increases up to $50 \mathrm{GW} / \mathrm{cm}^{2}$, distinct and elevated ripples are seen in Figure 2b. Their stability and smoothness also decreases. The protruding surface morphology shows sinusoidal behavior along with bead like structures. When the irradiance increases up to $75 \mathrm{GW} / \mathrm{cm}^{2}$ less distinct and narrow ripples are seen in Figure 2c. The periodicity does not change significantly. Another feature between the ripples is the remnants of turbulent melt flow. The regularity and periodicity of the ripples is enhanced at the highest irradiance of 100 $\mathrm{GW} / \mathrm{cm}^{2}$ shown in Figure $2 \mathrm{~d}$. Their appearance becomes less distinct. Interference between the incident beam and the scattered wave is one of the proposed mechanism for the formation of these ripples (Sipe et al., 1983). The average periodicity of the ripples varies from 20 to $30 \mu \mathrm{m}$ but it is much greater than the wavelength of the incident laser beam $(1.064$ $\mu \mathrm{m})$ for all irradiances. This suggests the exclusion of contribution of interference effects for the formation of these rippled structures. Certain hydrodynamical (instabilities) mechanisms may be more reasonably accounted for the growth of such kind of structures (Liu et al., 2011). Hydrodynamic expulsion is controlled by a competition between the inertial forces induced by the pressure field into the viscous melt and the surface tension that tends to maintain cohesion (Bleiner et al., 2006). These large scale ripple patterns look more like an unstable interface between the pool water and the wind blowing along its surface. The melted surface and expanding plasma plume above the target surface play the role of water and wind, respectively (Liu et al., 2011). These structures can also be considered as frozen morphology due to capillary waves (Young et al., 1984). The elevation of the ripples increases from $25 \mathrm{GW} / \mathrm{cm}^{2}$ to $50 \mathrm{GW} / \mathrm{cm}^{2}$. However, further increase in laser irradiance up to $100 \mathrm{GW} / \mathrm{cm}^{2}$ causes reduction in the elevation and clarity of the ripples. The initial increase is attributed to the enhanced energy deposition. When the laser irradiance increases above $50 \mathrm{GW} / \mathrm{cm}^{2}$ ripples become less distinct. This is explainable on the basis of shielding effect of plasma (Farid et al., 2012). When the energy deposition increases plume temperature and density also increases. This plume with species of high kinetic energies and high fluxes will be able to absorb incoming laser radiations and therefore less laser energy will be deposited on the zinc target. This shielding effect will be responsible for reducing the electron temperature and number density of zinc plasma as has been confirmed by the LIBS analysis (Section 2).

SEM images of Figures $3 \mathrm{a}-3 \mathrm{~d}$ present the inner boundaries of the laser ablated zinc. Cones and wave-like ridges are dominant structural features. Forward peaked cones with wide base and spherical top are seen at the surface

a)
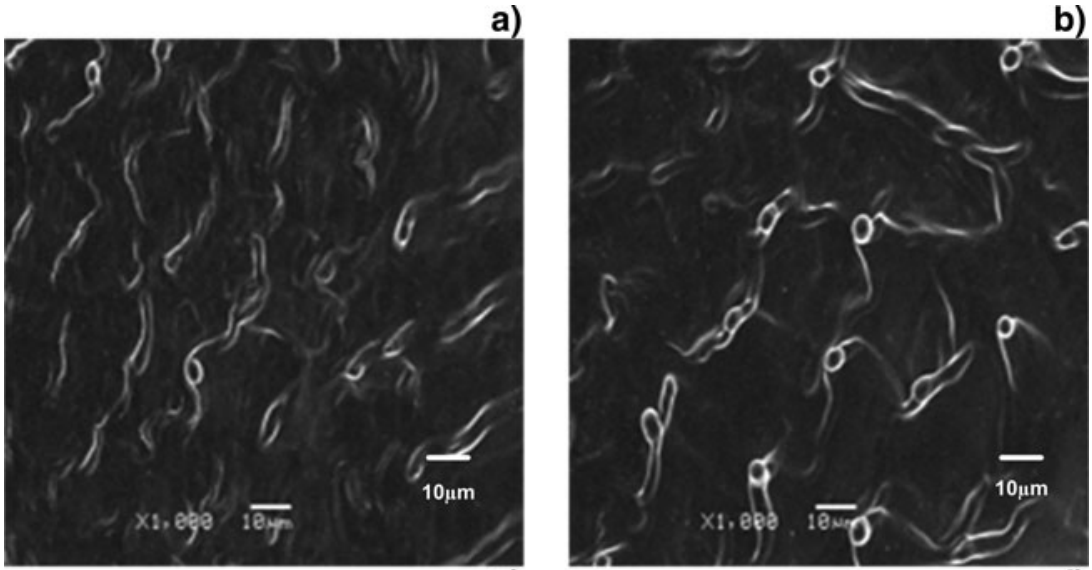

c)
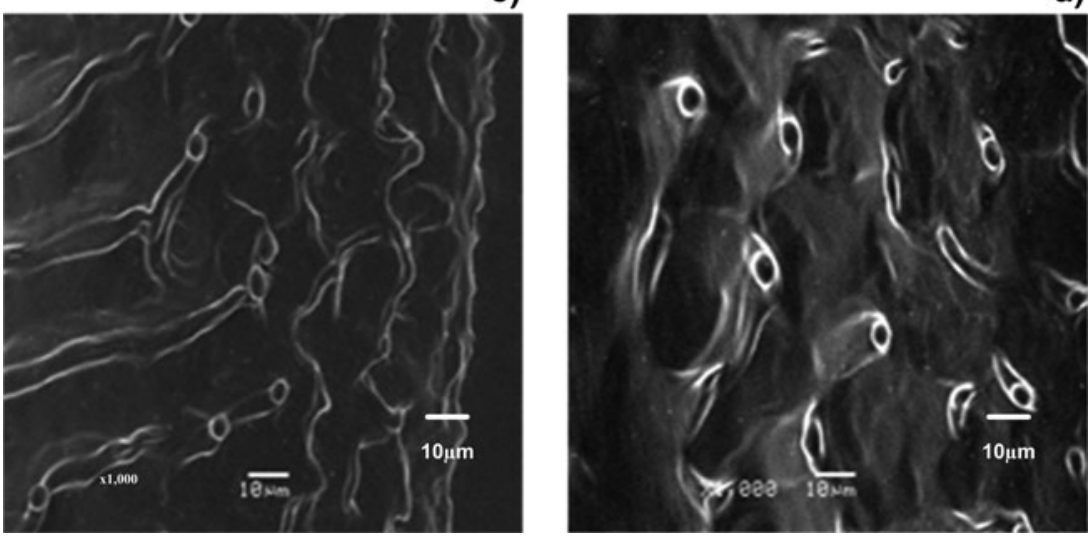

Fig. 3. SEM images of the inner boundaries of the ablated zinc targets at various laser irradiances of (a) 25, (b) 50, (c) 75, and (d) 100 $\mathrm{GW} / \mathrm{cm}^{2}$. 
irradiated with laser irradiance of $25 \mathrm{GW} / \mathrm{cm}^{2}$ as shown in Figure 3a. Preferential ablation due to evaporation resistive impurities, defects and hydrodynamic sputtering may be accounted for their formation (Kawakami et al., 2003; Dolgaev et al., 2006). Increasing the irradiance up to $50 \mathrm{GW} / \mathrm{cm}^{2}$ conical features are almost vanished and ridge formation is enhanced as shown in Figure 3b. Flakes are also formed in the inter ridged regions indicating the exfoliational sputtering. Further increase in irradiance up to $75 \mathrm{GW} / \mathrm{cm}^{2}$ increases the melted imprints in form of uplifted ridges and wavy patterns as shown in Figure 3c. Enhanced recoil pressure of the plasma splashes more material toward the boundary and causes ridge formation (Ko et al., 2007). The melt displacement and expulsion become do minanat (Cristoforetti et al., 2008). Melt pool perturbation may also lead to the growth of Rayleigh-Taylor instability resulting in the formation of these wavy patterns (Willis et al., 2000). When the irradiance increases to the $100 \mathrm{GW} / \mathrm{cm}^{2}$, cones with increased diameter, thick underlying ridges, and deep valleys are formed as shown in Figure 3d. Laser energy deposition at the boundary is smaller as compared to central ablated area because of the Gaussian distribution of the laser pulse. Shielding effects are therefore not dominant at peripheries due to lower irradiances (Cabalín et al., 1999; Bleiner, 2006). Hence, enhanced energy deposition with the increasing laser irradiance is responsible for the generation of these large size cones. The hydrodynamic pressure of the expanding plasma drives a shock wave toward the target surface in addition to the heating. The combined effect of heat and shock wave causes melting, evaporation, and sputtering of the target surface (Torrisi et al., 2003). SEM images of Figures $4 \mathrm{a}-4 \mathrm{~d}$ show the outer boundary region of ablated zinc targets. It comprises of the displaced material from the center and re-deposited plasma condensates in addition to the direct laser heating effects. The structures observed at outer boundary are cones, flakes, and cavities. The heating of subsurface layer and rapid volume expansion of absorbed gases in the underlying pores of the target surface lead to the formation of these cavities (Lappalainen et al., 1998). Explosive relaxation of the mechanical stresses in the superficial layer may also be accounted for the formation of these cavities (Tokarev et al., 2006). The size and number of cavities increase with the increase of laser irradiance up to a certain value and then decrease. It may be due to their re-filling with the melt.

\section{LIBS Analysis}

In order to correlate the ablation mechanism of irradiated zinc target with the plasma parameters, LIBS analysis was also

a)
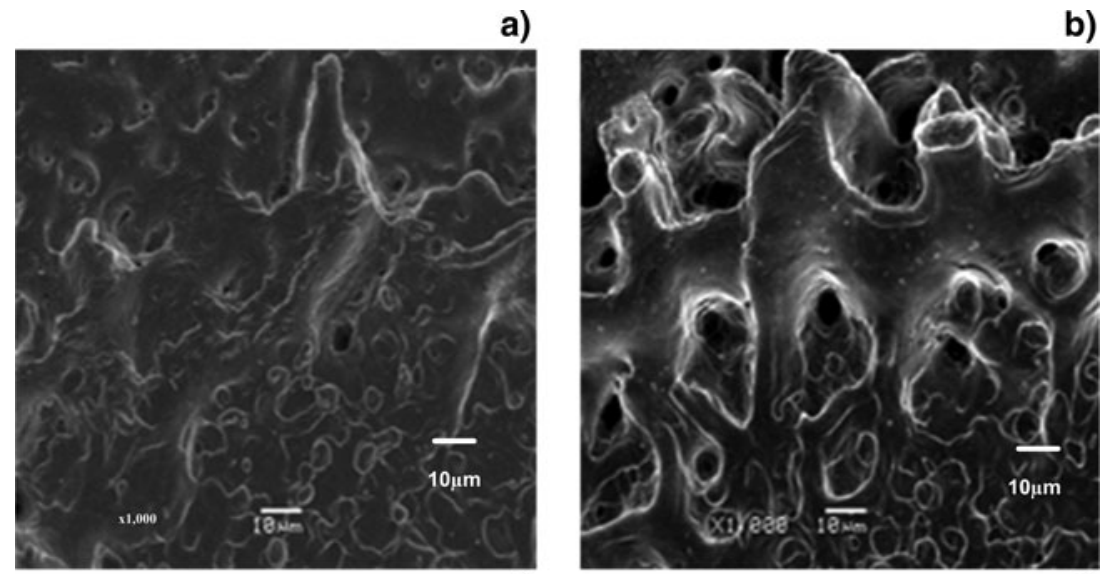

b)

c)
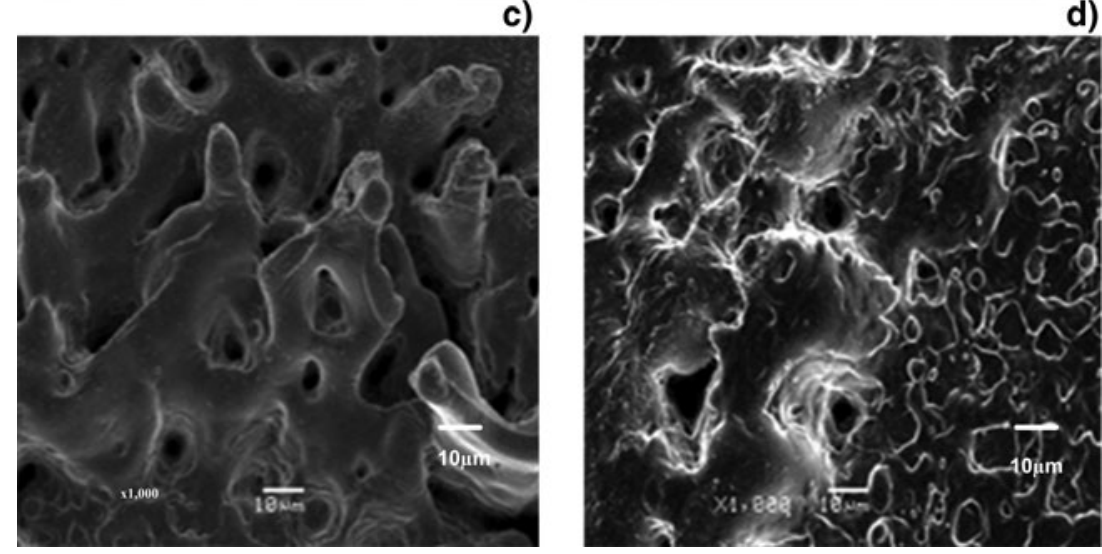

Fig. 4. SEM images of the outer boundaries of the ablated zinc targets at various laser irradiances of (a) 25, (b) 50, (c) 75, and (d) 100 $\mathrm{GW} / \mathrm{cm}^{2}$. 


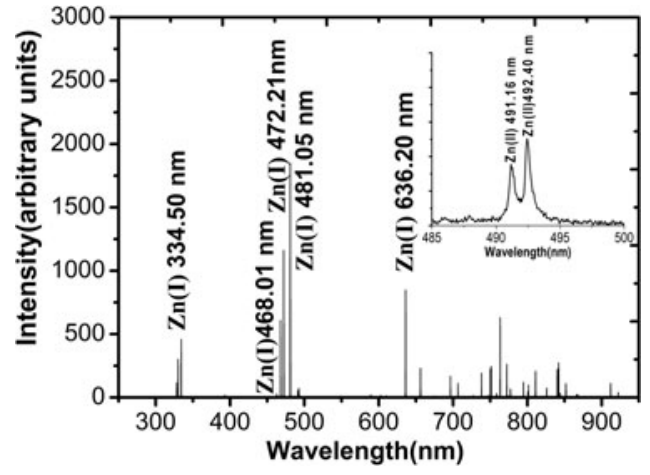

Fig. 5. The emission spectrum of laser induced zinc plasma under the ambient environment of Argon at a pressure of 20 Torr by using Nd:YAG laser $(\lambda=1064 \mathrm{~nm}, t=10 \mathrm{~ns})$ at irradiance of $63 \mathrm{GW} / \mathrm{cm}^{2}$.

performed. The plasma is characterized by its emission intensity, temperature, and number density of charged particles. All of these parameters are evaluated at various laser irradiances. Figure 5 shows the laser induced breakdown spectrum of zinc plasma obtained with $63 \mathrm{GW} / \mathrm{cm}^{2}$ irradiance in argon ambient at a pressure of 20 Torr. It contains different spectral lines from neutral zinc atoms at $334.5 \mathrm{~nm}, 468.0 \mathrm{~nm}$, $472.2 \mathrm{~nm}, 481.0 \mathrm{~nm}$, and $636.2 \mathrm{~nm}$ and from singly ionized zinc ions at $491.16 \mathrm{~nm}, 492.40 \mathrm{~nm}$ (shown in the inset) wavelengths. The rest of the lines correspond to $\operatorname{Ar}(\mathrm{I})$ at $696.54 \mathrm{~nm}, 763.51 \mathrm{~nm}, 810.37 \mathrm{~nm}, 811.53 \mathrm{~nm}$, etc., and indicate its breakdown down (Shaikh et al., 2006a). The obtained plasma is a mixture of ablated metal vapor and background gas.

Figure 6 shows the variation of emission intensity with the laser irradiance for the selected spectral lines corresponding to neutral zinc atoms. It depicts an initial increase of emission intensities with the increasing laser irradiance reaches a maximum then decreases followed by almost constant trend.

The obtained emission spectra are used to calculate the excitation temperature and number density of the zinc plasma. Few assumptions are made prior to the calculations which generally hold good for the characteristics of the

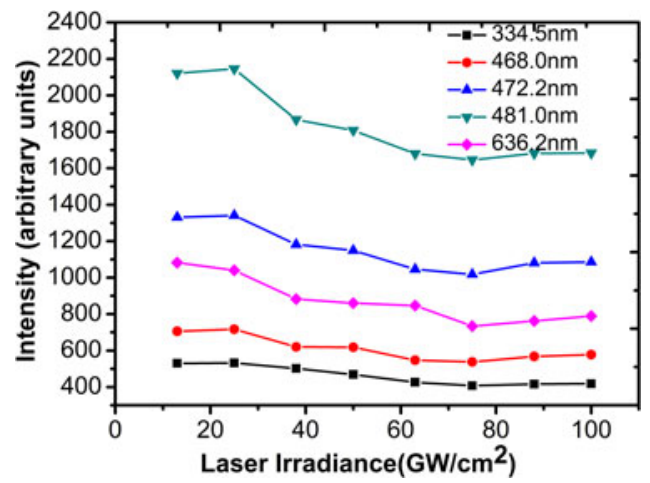

Fig. 6. (Color online) The variation of emission intensity of spectral lines of neutral zinc atoms at various laser irradiances ranging from $13 \mathrm{GW} / \mathrm{cm}^{2}$ to $100 \mathrm{GW} / \mathrm{cm}^{2}$ laser induced plasma in the concerned irradiance range. The plasma is considered to be in local thermal dynamical equilibrium (LTE) due to the dominance of collisional processes among the species and hence characterized by a homogenous temperature. The population in the excited states follows the Boltzmann distribution given by the following equation (Bashir et al., 2012a; Harilal et al., 1998; Iida et al., 1990; Cristoforetti et al., 2004; Hermann et al., 1993).

$$
\ln \left(\lambda_{\mathrm{mn}} I_{\mathrm{mn}} / g_{\mathrm{m}} A_{\mathrm{mn}}\right)=-E_{\mathrm{m}} / K T_{e}+\ln (N(T) / U(T)),
$$

where $\lambda_{\mathrm{mn}}, I_{\mathrm{mn}}, g_{\mathrm{m}}$, and $A_{\mathrm{mn}}$ are wavelength, intensity, statistical weight, and transition probability of the upper level state $\mathrm{m}$, respectively. On the right-hand side $U(T), N(T)$, $K, E_{\mathrm{m}}$, and $T_{\mathrm{e}}$ are partition function, total number density, Boltzmann constant, energy of the upper level state, and electron temperature, respectively. Boltzmann plot of the logarithmic term on the left-hand side versus the $E_{\mathrm{m}}$ gives a straight line whose slope is equal to the $-1 / K T_{\mathrm{e}}$ under the assumption made that the distribution is Boltzmann. The relevant spectroscopic parameters for the transitions of the zinc plasma used in the calculation are listed in Table 1 and have been taken from Shaikh et al. (2006; NIST database).

The transition lines at $334.5 \mathrm{~nm}\left(4 \mathrm{~s} 4 \mathrm{~d}^{3} \mathrm{D}_{3} \rightarrow 4 \mathrm{~s} 4 \mathrm{p}^{3} \mathrm{P}_{2}\right)$, $468.0 \mathrm{~nm}\left(4 \mathrm{~s} 5 \mathrm{~s}{ }^{3} \mathrm{~S}_{1} \rightarrow 4 \mathrm{~s} 4 \mathrm{p}{ }^{3} \mathrm{P}_{0}\right), 472.2 \mathrm{~nm}\left(4 \mathrm{~s} 5 \mathrm{~s}{ }^{3} \mathrm{~S}_{1} \rightarrow\right.$ $\left.4 \mathrm{~s} 4 \mathrm{p}{ }^{3} \mathrm{P}_{1}\right), 481.0\left(4 \mathrm{~s} 5 \mathrm{~s}{ }^{3} \mathrm{~S}_{1} \rightarrow 4 \mathrm{~s} 4 \mathrm{p}{ }^{3} \mathrm{P}_{2}\right)$, and 636.2(4s $4 \mathrm{~d}$ ${ }^{1} \mathrm{D}_{2} \rightarrow 4 \mathrm{~s} 4 \mathrm{p}{ }^{1} \mathrm{P}_{1}$ ) are used for the calculation of electron temperature.

The variation of electron temperature with laser irradiance is shown in Figure 7. It increases slightly from $6126 \mathrm{~K}$ to $6240 \mathrm{~K}$ with increasing laser irradiance from $25 \mathrm{GW} / \mathrm{cm}^{2}$ to $63 \mathrm{GW} / \mathrm{cm}^{2}$. With further increase in laser irradiance from $63 \mathrm{GW} / \mathrm{cm}^{2}$ to $75 \mathrm{GW} / \mathrm{cm}^{2}$ the electron temperature drops down from $6240 \mathrm{~K}$ to $6030 \mathrm{~K}$. With further increasing fluence up to a maximum value of $100 \mathrm{GW} / \mathrm{cm}^{2}$, the saturation with a negligible change (within error bar) in the electron temperature is achieved.

The plasma number density has been extracted from the profile of the line spectra obtained. Plasma species are under the influence of electric field of fast moving electron and slow ions. The perturbing electric field shifts the energy levels of the species leading to broadening called the Stark broadening. Other broadening mechanisms like Doppler and pressure broadening contribute little in this case and hence can be ignored. The relation of the full width at half maximum (FWHM) $\left(\Delta \lambda_{1 / 2}\right)$ of the stark broadened profile of the spectral lines and the electron number density of neon is given by the equation (Bashir et al., 2012a; Harilal et al., 1998; Iida et al., 1990; Cristoforetti et al., 2004; Hermann et al., 1993).

$$
N_{e}=\left(\Delta \lambda_{1 / 2} / 2 w\right) \times 10^{16} \mathrm{~cm}^{-3} .
$$

Where $w$ is the impact width parameter. The $\mathrm{Zn}(\mathrm{I})$ line at 636.23 is used to calculate the electron number density. 
Table 1. The spectroscopic data for the selected lines used to calculate the electron temperature and number density of laser induced zinc plasma (Sansonetti et al., 2005)

\begin{tabular}{|c|c|c|c|c|c|c|}
\hline $\begin{array}{l}\text { Wavelength } \\
\lambda(\mathrm{nm})\end{array}$ & Transitions & $\begin{array}{l}\text { Statistical } \\
\text { Weight } g_{m}\end{array}$ & $\begin{array}{c}\text { Transition } \\
\text { Probability } \mathrm{A}\left(\mathrm{s}^{-1}\right)\end{array}$ & $\begin{array}{l}\text { Relative Intensity } \\
\text { (arbitrary units) }\end{array}$ & $\begin{array}{l}\text { Energy of the Upper } \\
\text { level } E_{\mathrm{m}} \mathrm{cm}^{-1}\end{array}$ & $\begin{array}{c}\text { Energy of the Upper } \\
\text { level }(\mathrm{eV})\end{array}$ \\
\hline 334.502 & $4 \mathrm{~s} 4 \mathrm{~d}^{3} \mathrm{D}_{3} \rightarrow 4 \mathrm{~s} 4 \mathrm{p}^{3} \mathrm{P}_{2}$ & 7 & $1.70 \mathrm{e}+8$ & 800 & 62776.95 & 7.783 \\
\hline 468.014 & $4 \mathrm{~s} 5 \mathrm{~s}{ }^{3} \mathrm{~S}_{1} \rightarrow 4 \mathrm{~s} 4 \mathrm{p}{ }^{3} \mathrm{P}_{0}$ & 3 & $1.55 \mathrm{e}+7$ & 300 & 53672.24 & 6.654 \\
\hline 472.215 & $4 \mathrm{~s} 5 \mathrm{~s}{ }^{3} \mathrm{~S}_{1} \rightarrow 4 \mathrm{~s} 4 \mathrm{p}^{3} \mathrm{P}_{1}$ & 3 & $4.58 \mathrm{e}+7$ & 400 & 53672.24 & 6.654 \\
\hline 481.053 & $4 \mathrm{~s} 5 \mathrm{~s}{ }^{3} \mathrm{~S}_{1} \rightarrow 4 \mathrm{~s} 4 \mathrm{p}^{3} \mathrm{P}_{2}$ & 3 & $7.00 \mathrm{e}+7$ & 400 & 53672.24 & 6.654 \\
\hline 636.234 & $4 \mathrm{~s} 4 \mathrm{~d}^{1} \mathrm{D}_{2} \rightarrow 4 \mathrm{~s} 4 \mathrm{p}^{1} \mathrm{P}_{1}$ & 5 & $4.74 \mathrm{e}+7$ & 1000 & 62458.51 & 7.744 \\
\hline
\end{tabular}

The self-absorption for the spectral line $636.23 \mathrm{~nm}$ has been considered. The selected line must be sharp and the least broaden for fulfilling the criteria of minimum selfabsorption. The selected line to be used for the calculations of number density is $632.23 \mathrm{~nm}$ for $\mathrm{Zn}(\mathrm{I})$ and it is sharp and the least broaden line as compared to all other lines of the obtained spectra. In order to check the self-absorption (SA) of a spectral line, following relation can be utilized for the evaluation of the parameter of SA (Cristoforetti et al., 2010b):

$$
\mathrm{SA}=\left(\Delta \lambda / 2 w_{s} N_{e}\right)^{1 / \alpha}
$$

Where $\alpha=-0.54$. The $\Delta \lambda, w_{\mathrm{s}}$, and $N_{\mathrm{e}}$ is experimentally determined stark width, half width stark broadening parameter, and electron number density calculated from the $H_{\alpha}$ line as (Sherbini et al., 2005):

$$
n_{e}\left(\mathrm{~cm}^{-3}\right)=C(\lambda, T)(\Delta \lambda)^{3 / 2}
$$

where $\Delta \lambda$ is FWHM of $H_{\alpha}$ line and $C(\lambda, T)$ is a coefficient weakly dependent on density and temperature and has been tabulated in Griem et al. (1964). The value for $\Delta \lambda$ is obtained by fitting the line with a Voigt profile and by considering the width of its Lorentzian component (Cristoforetti et al., $2010 b)$. The Stark broadening parameter for $636.23 \mathrm{~nm}$ $\mathrm{Zn}(\mathrm{I})$ line has been taken from the work reported in Sherbini

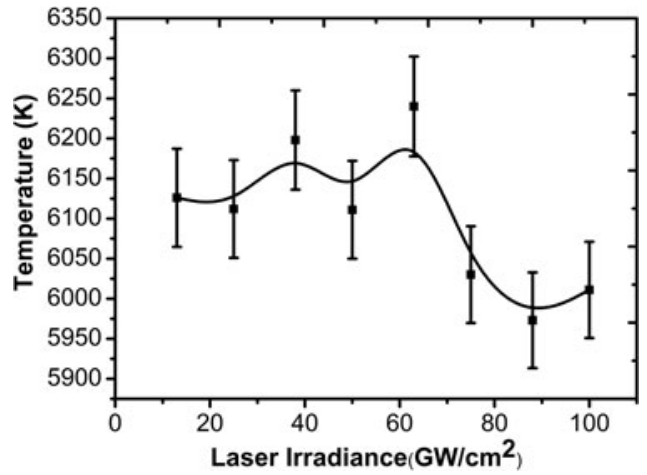

Fig. 7. The variation of electron temperature of laser induced zinc plasma obtained at various laser irradiances ranging from $13 \mathrm{GW} / \mathrm{cm}^{2}$ to $100 \mathrm{GW} / \mathrm{cm}^{2}$. et al. (2013). When SA value is nearly equal to 1 and it indicates that self-absorption effects are negligible while $\mathrm{SA} \ll 1$ indicates that nonlinear effects are strong (Cristoforetti et al., $2010 b$ ). By putting data from our experiment the evaluated value of SA comes out to be nearly equal to one and therefore indicates that self-absorption is negligible for our case.

The self-absorption coefficient can also be calculated as (Sherbini et al., 2012):

$$
\mathrm{SA}=I\left(\lambda_{0}\right) / I_{0}\left(\lambda_{0}\right)=\left(\Delta \lambda_{1} / \Delta \lambda_{0}\right)^{1 / \alpha}=\left(n_{e}^{1} / n_{e}^{H \alpha}\right)^{1 / \alpha} .
$$

Where $I\left(\lambda_{0}\right)$ and $I_{0}\left(\lambda_{0}\right)$ are the spectral intensity of line in limit of negligible self-absorption and experimentally measured line height respectively. $\Delta \lambda_{0}$ and $\Delta \lambda_{1}$ are the Lorentzian component of spectral line if the line is optically thin and broadened FWHM of Lorentzian component of the same line because of self-absorption process respectively. $n_{\mathrm{e}}^{1}$ and $n_{\mathrm{e}}^{H \alpha}$ are the number densities evaluated from line under investigation and $H_{\alpha}$ line, respectively. The obtained spectral line is plotted and fitted with the Lorentizan profile to obtain the FWHM and $w$ (impact width parameter).

The variation of electron number density with increasing laser irradiance is shown in Figure 8. It increases from $1.3586 \times 10^{18} \mathrm{~cm}^{-3}$ to $1.4433 \times 10^{18} \mathrm{~cm}^{-3}$ with increasing laser irradiance from $25 \mathrm{GW} / \mathrm{cm}^{2}$ to $63 \mathrm{GW} / \mathrm{cm}^{2}$. With further increase in laser irradiance from $63 \mathrm{GW} / \mathrm{cm}^{2}$ to $75 \mathrm{GW} / \mathrm{cm}^{2}$ it drops down slightly to a value of $1.4296 \times$ $10^{18} \mathrm{~cm}^{-3}$. With further increasing fluence up to a maximum

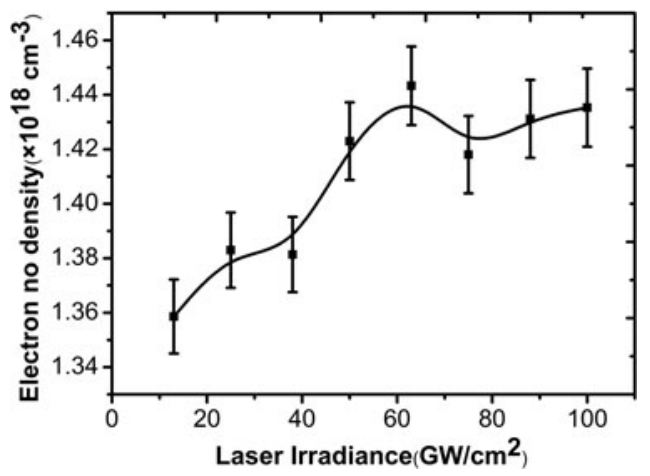

Fig. 8. The variation of electron number density of zinc plasma at various laser irradiances ranging from $13 \mathrm{GW} / \mathrm{cm}^{2}$ to $100 \mathrm{GW} / \mathrm{cm}^{2}$. 
value of $100 \mathrm{GW} / \mathrm{cm}^{2}$, the saturation with a negligible change (within error bar) in the electron density is achieved.

The observed trend in electron temperature and number density is divided into three regions: an initial increase with the increase of laser irradiance reaches a maximum at $63 \mathrm{GW} / \mathrm{cm}^{2}$ in the first region, then decrease in the second region, and finally saturation is achieved in the third region. This can be explained as follows: when a nanosecond laser interacts with the material it heats up the target until its melting point is reached and eventually causes it to eject into the form of vapor (Farid et al., 2013). Initially, at lower irradiances the ablation rate increases with the increase of laser irradiance which in turn increases the temperature and charge particle density in the plasma (Luo et al., 2010). The background pressure also effectively confines the plasma species which further adds to increase the temperature and density of charged particle by reducing its expansion rate. The background argon ambient at a pressure of 20 Torr is seemed to be sufficient to confine the generated target vapors. Plasma reaches its maximum temperature and number density at $63 \mathrm{GW} / \mathrm{cm}^{2}$. This vapor plume continues to absorb the laser energy through inverse-bremsstrahlung and direct photo ionization processes and start to shield the target from the laser irradiation (Harilal et al., 1998). Further increase in the irradiance reduces energy deposition to the target due to enhanced shielding effect of the plasma which leads to decrease the electron temperature and number density. At still higher irradiances the saturation region is observed due to the formation of a self-regulating regime in addition to the shielding effect (Cristoforetti et al., 2008; Bleiner et al., 2006). In this regime, the density, temperature, and dimensions of plume adjust in such a way that plasma absorb the same amount of laser energy to maintain the self-regulating regime (Harilal et al., 1998; Hafeez et al., 2008).

Cristoforetti et al. (2010a) has addressed the criteria for the establishment of LTE in addition to the McWhirter criterion. The McWhirter criterion is a necessary but not the sufficient condition to ensure the existence of LTE in plasma. The condition of LTE has been assessed for plasma in three states namely stationary and homogenous, transient and homogeneous, and transient and inhomogeneous. Considering our plasma as transient and homogenous, we have fulfilled the criteria for LTE by evaluating the following two conditions: (1) The McWhirter criteria $n_{\mathrm{e}}\left(\mathrm{cm}^{-3}\right)=1.6 \times 10^{12}$ $(T)^{1 / 2}\left(\Delta E_{\mathrm{nm}}\right)^{3}$ has been fulfilled (Cristoforetti, 2010). For the excitation temperature $T_{\mathrm{e}}=6240 \mathrm{~K}$, the number density of neon comes out to be of the order of $10^{14}$. (2) For homogenous and transient plasma, if the time needed for the establishment of excitation and ionization equilibrium (relaxation time) is much shorter than the time for variation of thermodynamic parameters (mainly neon) then the plasma evolves through quasi-equilibrium near LTE states (Cristoforetti et al., 2010a). The rate coefficient for the collisional excitation from ground to first excited state of the resonance series being the slowest one has been considered for the rough estimation of this relaxation time. This can be expressed as (Cristoforetti et al., 2010a):

$$
\begin{aligned}
t_{\text {rel }}=1 / n_{e}<v a_{12}> & =6.3 \times 10^{14} \Delta E_{21}(K T)^{1 / 2} \\
& \exp \left(\Delta E_{21} / K T\right) / n_{e} f_{12}<g>.
\end{aligned}
$$

Where $t_{\text {rel }}$ is relaxation time, $\Delta E_{21}$ and $K T$ are both expressed in $\mathrm{eV}$. The $f_{12}$ is the transition oscillator strength, $\langle g\rangle$ is effective gaunt factor and $\mathrm{n}_{\mathrm{e}}$ is number density in $\mathrm{cm}^{-3}$. The calculated time (of the order of $10^{-9} \mathrm{~s}$ ) came out to be reasonably shorter than the typical decay time of electron temperature and number density (approximately $1 \mu \mathrm{s}$ ).

LIBS analysis can be well correlated with the SEM investigations. The initial increase in $T_{\mathrm{e}}$ and $n_{\mathrm{e}}$ with the increasing laser irradiance is responsible for the growth of distinct and well defined ripples. After obtaining the maxima, $T_{\mathrm{e}}$ and $n_{\mathrm{e}}$ decrease because of shielding effect and formation of selfregulatory region. Due to less energy deposition of electrons to the lattice the grown structures become diffusive and narrow.

\section{CONCLUSIONS}

The effect of various laser irradiances (ranging from $13 \mathrm{GW} / \mathrm{cm}^{2}$ to $100 \mathrm{GW} / \mathrm{cm}^{2}$ ) on surface morphology and plasma parameters of zinc have been investigated. SEM investigation revealed the formation of rippled structures at the center, whereas cones and cavities are seen at the boundary of ablated zinc targets. Initially with the increase of laser irradiance distinctness of the ripples increases due to enhanced energy deposition. They become less pronounced with the further increase in irradiance due to enhanced shielding effect. Due to the Gaussian distribution of laser pulse, the energy deposited at boundary is smaller as compared to the center. Hence the shielding effect is not produced at the boundaries and energy deposition continues to increase with the increase of laser irradiance. This results in the growth of large size structures and enhanced melting effects at the inner and outer boundary regions respectively at high irradiances. This trend is well correlated with the LIBS results in which an initial increase in the electron temperature and density with the increase of laser irradiance up to a $63 \mathrm{GW} / \mathrm{cm}^{2}$ and then a decrease and saturation is observed at high irradiances.

\section{REFERENCES}

Al-Sherbini, A.M., Al-Sherbini, Th.M., Hegazy, H., Cristoforetti, G., Legnaioli, S., Palleschi, V., Pardini, L., Salvetti, A. $\&$ Tognoni, E. (2005). Evaluation of self-absorption coefficients of aluminium emission lines in laser-induced breakdown spectroscopy measurements. Spectrochim. Acta Part B 60, 1573-1579.

Bashir, S., Farid, N., Mahmood, M. \& Rafique, M.S. (2012a). Influence of ambient gas and its pressure on the laser-induced 
breakdown spectroscopy and the surface morphology of laserablated Cd. Appl. Phys. A 107, 203-212.

Bashir, S., Rafique, M.S. \& Husinsky, W. (2012b). Femtosecond laser-induced subwavelength ripples on $\mathrm{Al}, \mathrm{Si}, \mathrm{CaF}_{2}$ and CR-39. Nucl. Instrum. Meth. B. 275, 1-6.

BäUeRLE, D. 2011. Laser Processing and Chemistry. Heidelberg: Springer-Verlag.

Bleiner, D. \& Bogaerts, A. (2006). Multiplicity and contiguity of ablation mechanisms in laser-assisted analytical microsampling. Spectrochimica Acta Part B 61, 421-432.

Butt, M.Z., Dilawar, A., Naseem, S., Bashir, F. \& Ishtiaq, M. (2013). Surface morphology and structural characterization of high-purity iron irradiated with Nd:YAG pulsed laser. Physica $B$ 425, 58-65.

Cabalín, L.M., Romero, D., Baena, J.M. \& Laserna, J.J. (1999). Saturation effects in the laser ablation of stainless steel in air at atmospheric pressure. Fresenius J. Anal. Chem. 365, 404-408.

Chamorro, J.C., Uzuriaga, J. \& Riascos, H. (2012). Laser induced aluminiun plasma analysis by optical emission spectroscopy in a nitrogen background gas. J. Appl. Phys. Conf. Ser. 370, 1-6.

Chen, M., Liu, Xiangdong., Liu, Yuehua. \& Zhao, Mingwen. (2012a). Characterization of regular periodic surface structure by multi-pulse laser irradiation of a Zinc target. Chin. Opt. Lett. 10, 051402.

Chen, M., Liu, Y.H., Liu, X.D. \& Zhao, M.W. (2012b). Role of lens to sample distance during laser-induced damage in zinc targets. Laser Phys. Lett. 9, 730-733.

Chen, Z., Wu, Qiang., Yang, M., Baiquan, T., Yao, Jianghong, Rupp, Romano A., CAO, YAAN \& JingJun, X. (2012c). Generation and evolution of plasma during femtosecond laser ablation of silicon in different ambient gases. Laser Part. Beams 13, 1-7.

Cristoforetti, G., Giacomo, A.D., Dell'Aglio, M., Legnaioli, S., Tognoni, E., Palleschi, V. \& Omenetto, N. (2010a). Local thermodynamic equilibrium in laser-induced breakdown spectroscopy: Beyond the McWhirter criterion. Spectrochimica Acta Part B 65, 86-95.

Cristoforetti, G., Legnaioli, S., Palleschi, V., Tognoni, E. \& BenEDETTI, P.A. (2008). Observation of different mass removal regimes during the laser ablation of an aluminium target in air. J. Anal. At. Spectrom. 23, 1518-1528.

Cristoforetti, G., Lorenzetti, G., Legnaioli, S. \& Palleschi, V. $(2010 b)$. Investigation on the role of air in the dynamical evolution and thermodynamic state of a laser-induced aluminium plasma by spatial- and time-resolved spectroscopy. Spectrochimica Acta Part B 65, 787-796.

Cristoforetti, G., Palleschi, V., Salvetti, A. \& Tognoni, E. (2004). Influence of ambient gas pressure on laser-induced breakdown spectroscopy technique in the parallel double-pulse configuration. Spectrochimica Acta B 59, 1907-1917.

Crouch, C.H., Carey, J.E., Warrender, J.M., Mazur, E. \& Genin, F.Y. (2004). Comparison of structure and properties of femtosecond and nanosecond laser-structured silicon. Appl. Phys. Lett. 84, 1850-1852.

Dolgaev, S.I., Fernández-Pradas, J.M., Morenza, J.L., Serra, P. $\&$ ShafeEv, G.A. (2006). Growth of large microcones in steel under multipulsed Nd:YAGlaser irradiation. Appl. Phys. A. 83, 417-420.

El Sherbini, M.A., Аboulfotouh, A.N., Rashid, F., Allam, S.H., Al-Kaoud, A.M., Dakrouri, A.E. \& El. Sherbini, T.M. (2013). Spectroscopic measurement of Stark broadening parameter of the $636.2 \mathrm{~nm}$ Zn I-line. Nat. Sci. 5, 501-507.
Farid, N., Bashir, S. \& Khaliq, M. (2012). Effect of ambient gas conditions on laser-induced copper plasma and surface morphology. Phys. Scr. 85, 015702.

Farid, N., Cong, L., Hongbei, W.E. \& Hongbin, D. (2013). Laserinduced breakdown spectroscopic characterization of tungsten plasma using the first, second, and third harmonics of an Nd:YAG laser. J. Nucl. Mater. 433, 80-85.

GrIEM, H.R. (1964). Plasma Spectroscopy.

Hafeez, S., Shaikh, N.M., Rashid, B. \& Baig, M.A. (2008). Plasma properties of laser-ablated strontium target. J. Appl. Phy. D. 3, 083117.

Hanif, M., SAlik, M., Sheikh, Nek M. \& Baig, M.A. (2013). Laserbased optical emission studies of barium plasma. Appl. Phys. B 110, 563-571.

Harilal, S.S., Bindhu, C.V., Nampoori, V.P.N. \& Vallabhan, C.P.G. (1998). Temporal and Spatial behavior of electron density and temperature in a laser -produced plasma from $\mathrm{YBa}_{2} \mathrm{Cu}_{3} \mathrm{O}_{7}$. Appl. Spect. 52, 449-455.

Hermann, J., Boulmer-Leborgne, C., Dubreuil, B. \& Mihailescu, I.N. (1993). Influence of irradiation conditions on plasma evolution in laser surface interaction. J. Appl. Phys. 74, 3071.

IIDA, Y. (1990). Effects of atmosphere on laser vaporization and excitation processes of solid samples. Spectrochim. Acta 45B, 1353-1367.

Ilyas, B., Dogar, A.H., Ullah, S., Mahmood, N. \& Qayyum, A. (2012). Multiply charged ion emission from laser produced tungsten plasma. Laser Part. Beams 30, 651-657.

Jelani, M., Bashir, S., Rehman, M.K., Ahamad, R., Haq, F., Yousaf, D., Akram, M., Afzal, N., Chaudhry, M.U., Mahmood, K., Hayat, A. \& Ahmad, S. (2013). Effect of laser fluence on surface, structural and mechanical properties of $\mathrm{Zr}$ after irradiation in the ambient environment of oxygen. Eur. Phys. J. D. 159, 1-7.

Juan, X.U., Min, H.U., Ying, T.X. \& KuI, L.C. (2013). Zinc ion implantation-deposition modification of titanium for enhanced adhesion of focal plaques of osteoblast-like cells. Chin. Med. J. 126, 3557-3560.

KaWakami, Y. \& Ozawa, E. (2003). Tungsten microcone growth by laser irradiation. Appl. Surf. Sci. 218, 175-187.

Khan, S., Bashir, S., Hayat, A., Rahman, M.K. \& Haq, F.U. (2013). Laser-induced breakdown spectroscopy of tantalum plasma. Phys. Plasmas 20, 073104.

Ko, S.H., Pan, H., Hwang, D.J., Chung, J., Ryu, S. \& Costas, P. (2007). High resolution selective multilayer laser processing by nanosecond laser ablation of metal nanoparticle films. J. Appl. Phys. 102, 093102.

Lappalainen, J., Frantti, J. \& Lantto, V. (1998). Particulate Formation in PZT Thin Films during Pulsed Laser Ablation Deposition. J. Korean Phys. Soc. 32, 1183-1186.

Latif, A., Bhatti, K.A., Rahman, M.K. \& Rafique, M.S. (2012). Effect of UV irradiation on the structural, optical and electrical properties of platinum. Rad. Eff. \& Def. in Sol. 167, 929-936.

LiU, Y., JiAnG, M. Q., YANG, G.W., GuAn, Y.J. \& DAI, L.H. (2011). Surface rippling on bulk metallic glass under nanosecond pulse laser ablation. Appl. Phys. Lett. 99, 91902.

Luo, W.F., ZhaO, X.X., Sun, Q.B.,GaO, C.X., TANG, J., Wang, H.J. \& Zhно, W. (2010). Characteristics of the aluminum alloy plasma produced by a $1064 \mathrm{~nm} \mathrm{Nd:YAG} \mathrm{laser} \mathrm{with} \mathrm{different}$ irradiances. Pramana -J. Phys. 74, 945-959. 
NIST Atomic Spectra Database. http://physics.nist.gov Kurucz ouput In Atomic Spectral line database from R.L.Kurucz,s CC ROM 23 Ed.),. http://physics.nist.gov Kurucz ouput

Sarkar, A., Shah, R.V., Alamelu, D. \& Aggarwal, S.K. (2011). Studies on the ns-IR-laser-induced plasma parameters in the vanadium oxide. J. Atom. Mol. \& Opt Phys 2011, 1-8.

Shaikh, N.M., Rashid, B., Hafeez, S., Jamil, Y. \& Baig, M.A. (2006a). Measurement of electron density and temperature of a laser-induced zinc plasma. J. Phys. D. 39, 384-1391.

Shaikh, N.M., Rashid, B., Hafeez, S., Mahmood, S., Saleem, M. \& BAIG, M.A. (2006b). Diagnostics of cadmium plasma produced by laser ablation. J. Appl. Phys. 100, 073102.

Sherbini, E.A.M. \& SAAD Al Aamer, A.A. (2012). Measurement of plasma parameters in laser-induced breakdown spectroscopy using Si-lines. World J. Nano. Sci. Eng. 2, 206-212.

Sipe, J.E., Young, J.F., Preston, J.S. \& VAn Driel, H.M. (1983). Laser-induced periodic surface structure. I. Theory. Phys. Rev. B 27, 1141-1154.

Sobhani, M. \& Mahdieh, M.H. (2013). Comparison of sub-micro/ nano structure formation on polished silicon surface irradiated by nanosecond laser beam in ambient air and distilled water. Laser Part. Beams 30, 1-9.

TOKAREV, V.N. (2006). Viscous liquid expulsion in nanosecond UV laser ablation: from "clean" ablation to nanostructures. Laser Phys. 16, 1291-1307.

Torrisi, L., Gammino, S., Mezzasalma, A.M., Gentile, C., Krása, J., LÁska, L., Rohlena, K., BADZiaK, J., PARYs, P. \& WolowsKi, J. (2003). Tantalum irradiation by high power pulsed laser at 1315 and $438 \mathrm{~nm}$ wavelengths. Appl. Surf. Sci. 220, 193-202.

Trtica, M., Batani, D., Redaelli, R., Limpouch, J., Kmetik, V., Ciganovic, J., Stasic, J., Gakovic, B. \& Momcilovic, M. (2013). Titanium surface modification using femtosecond laser with $1013-1015 \mathrm{~W} / \mathrm{cm}^{2}$ intensity in vacuum. Laser Part. Beams 31, 29-36.

Willis, D.A. \& Xu, X. (2000). Transport phenomena and droplet formation during pulsed laser interaction with thin films. J. Heat Trans. 122, 763.

Young, J.F., SiPE, J.E. \& VAN Driel, H.M. (1984). Laser-induced periodic surface structure. III. Fluence regimes, the role of feedback, and details of the induced topography in germanium. Phys. Rev. B 30, 2001-2015. 